\title{
Caught Red-Handed
}

The approach to clinical conundrums by an expert clinician is revealed through the presentation of an actual patient's case in an approach typical of a morning report. Similarly to patient care, sequential pieces of information are provided to the clinician, who is unfamiliar with the case. The focus is on the thought processes of both the clinical team caring for the patient and the discussant.

This icon represents the patient's case. Each paragraph that follows represents the discussant's thoughts.

\author{
Valerie M. Vaughn, $\mathrm{MD}^{{ }^{1}, 2}$, Sean J. Callahan, $\mathrm{MD}^{3}$, James Pile, $\mathrm{MD}^{4}$, Powel Kazanjian, MD ${ }^{1}$, Andrew Odden, MD ${ }^{5}$
}

${ }^{1}$ Department of Internal Medicine, University of Michigan, Ann Arbor, Michigan; ${ }^{2}$ The Patient Safety Enhancement Program, University of Michigan and VA Ann Arbor Health System, Ann Arbor, Michigan; ${ }^{3}$ Department of Internal Medicine, University of Virginia Health System, Charlottesville, Virginia; ${ }^{4}$ Medicine Institute, Cleveland Clinic, Cleveland, Ohio; ${ }^{5}$ Department of Medicine, Washington University School of Medicine, St. Louis, Missouri.

A previously healthy 58 -year-old man presented to a community hospital's emergency department 1 day after the sudden onset of a severe headache, fever, diffuse abdominal pain, nausea, vomiting, and disorientation. The patient had a history of allergic rhinitis and his only medication was a daily multivitamin.

Key features of this patient's presentation include the abrupt onset of severe headache, disorientation, fever, and abdominal pain. The list of entities likely to make a previously healthy individual this ill this quickly is typically circumscribed. His presentation raises the possibility of bacterial meningitis (including Listeria, given his age), viral encephalitis, or other extraneural etiologies of sepsis. Noninfectious explanations seem much less likely given the rapid tempo of illness.

He lived in the upper Midwestern United States and denied any recent travel outside of the region. His family reported he had recently seen a tick on his clothing but had not noticed a bite. He worked in a beer-bottling plant, was an avid gardener, and owned a dog. He had no history of tobacco, alcohol, or illicit drug abuse.

His proclivity for gardening and apparent tick exposure raise the question of tick-borne illnesses. This would constitute a rather explosive onset for any of these; however, babesiosis, Rocky Mountain spotted fever (RMSF), ehrlichiosis, and anaplasmosis could present this abruptly, with dog exposure linked to RMSF.

${ }^{2}$ On physical examination, his temperature was $40.7^{\circ} \mathrm{C}$, heart rate was 115 beats per minute, respiratory rate was 16 breaths per minute, and blood pressure was 92/45 mm Hg. Pulse oximetry was $98 \%$ on ambient air. He was disoriented to place and situation, and somnolent but arousable with stimulation. Cardiopulmonary exam was notable for tachycardia. Abdominal exam revealed diffuse tenderness

*Address for correspondence and reprint requests: Valerie M. Vaughn, MD, 3119 Taubman Center, 1500 E. Medical Center Dr., SPC 5376, Ann Arbor, Ml 48109-5376; E-mail: valmv@umich.edu; Phone: 734-647-1599; Fax: 734-232-9343

Additional Supporting Information may be found in the online version of this article.

Received: November 6, 2015; Revised: February 19, 2016; Accepted: February 20, 2016

2016 Society of Hospital Medicine DOI 10.1002/jhm.2583

Published online in Wiley Online Library (Wileyonlinelibrary.com). without rebound or guarding. His spleen was palpable just below the left costal margin. Skin examination revealed an erythematous, morbilliform rash covering his entire body including his palms and soles. Pupils were equal, round, and reactive to light. Reflexes were symmetric and $2+$ throughout, and the remainder of his neurologic exam was normal. There was no nuchal rigidity.

The potential causes of fever and rash are myriad, although the severity and acuity of this patient's illness narrow the differential considerably, likely to an infectious cause. Diagnoses that typically include a generalized exanthem involving the palms and soles are meningococcal meningitis, overwhelming Staphylococcus aureus sepsis, RMSF (realizing that this disease is not common in the upper Midwest), and toxic shock syndrome. The rash described is not the classic and/or fully developed rash typical of any of these; subsequent evolution to a petechial appearance would lend further support to the first 3 diagnoses. Ehrlichiosis is still a possibility, although the palm and sole involvement would be unusual. The presence of a rash makes anaplasmosis very unlikely, although not entirely excluded. The finding of modest splenomegaly does not help further distinguish between these possibilities.

Empiric antimicrobials should be immediately administered after blood cultures, a complete blood count, and coagulation studies are obtained. Doxycycline would be appropriate to treat the possible tick-borne diseases already mentioned, whereas antimicrobials appropriate to cover communityacquired bacterial meningitis in a 58-year-old (ie, vancomycin, ampicillin, and a third-generation cephalosporin) should also be empirically administered. Given the patient's altered mentation, a brain computed tomography (CT) should be urgently obtained. Provided this did not show evidence of increased intracranial pressure and that coagulation studies and a platelet count did not suggest a contraindication, a lumbar puncture should then be performed promptly. The patient should be placed in droplet precautions until meningococcal disease is excluded. Although most patients with bacterial meningitis will exhibit meningismus, a substantial minority will not.

The white blood cell count was $13,300 / \mathrm{mm}^{3}$ with $84 \%$ neutrophils, $5.6 \%$ lymphocytes, and $5 \%$ monocytes. The hemoglobin was $13.6 \mathrm{~g} / \mathrm{dL}$ and the platelet count was $86,000 / \mathrm{mm}^{3}$. Serum sodium was $137 \mathrm{mmol} / \mathrm{L}$, potassium $4.2 \mathrm{mmol} / \mathrm{L}$, chloride $104 \mathrm{mmol} / \mathrm{L}$, bicarbonate $22 \mathrm{mmol} / \mathrm{L}$, 
blood urea nitrogen $29 \mathrm{mg} / \mathrm{dL}$, creatinine $1.08 \mathrm{mg} / \mathrm{dL}$ (baseline $0.8 \mathrm{mg} / \mathrm{dL}$ ) and glucose $123 \mathrm{mg} / \mathrm{dL}$. Total protein was $4.7 \mathrm{~g} / \mathrm{dL}$ (normal 6.0-8.3 g/dL), albumin $2.5 \mathrm{~g} / \mathrm{dL}$ (normal 3.5-4.9 g/dL), aspartate aminotransferase $68 \mathrm{IU} / \mathrm{L}$ (normal 8-30 IU/L), alanine aminotransferase $68 \mathrm{IU} / \mathrm{L}$ (normal 7-35 IU/L), alkaline phosphatase 106 IU/L (normal 30-130 IU/ $\mathrm{L}$ ), and total bilirubin $0.5 \mathrm{mg} / \mathrm{dL}$ (normal $0.2-1.2 \mathrm{mg} / \mathrm{dL}$ ). Troponin was $0.84 \mathrm{ng} / \mathrm{mL}$ (normal $<0.3 \mathrm{ng} / \mathrm{mL}$ ). C-reactive protein was $24.2 \mathrm{mg} / \mathrm{dL}$ (normal $0.0-0.6 \mathrm{mg} / \mathrm{dL}$ ) and erythrocyte sedimentation rate was $30 \mathrm{~mm}$ (normal 0-15 mm).

These laboratory results do not significantly affect the differential diagnosis. Although nonspecific, moderate thrombocytopenia and modest elevation of hepatic transaminases are typical for tick-borne diseases, whereas leukocytosis is somewhat atypical for these entities. Marked elevation of the Creactive protein with a less striking increase in the erythrocyte sedimentation rate, along with significant hypoalbuminemia, are commonly encountered early in the course of critical infectious illnesses. The elevated troponin likely reflects severe sepsis and demand ischemia, and is associated with a less favorable prognosis; an electrocardiogram and serial cardiac biomarkers are appropriate to help exclude an acute coronary syndrome. As already noted, blood cultures need to be obtained and a lumbar puncture should be performed, provided this can be safely accomplished.

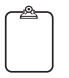
CT of the head was normal. A lumbar puncture was performed. Cerebrospinal fluid was acellular with a protein level of $58 \mathrm{mg} / \mathrm{dL}$ (normal $<45 \mathrm{mg} / \mathrm{dL}$ ). Blood, urine, and cerebrospinal fluid cultures were obtained. An electrocardiogram demonstrated sinus tachycardia without signs of ischemia, and a transthoracic echocardiogram showed normal ventricular function. CT of the chest, abdomen, and pelvis revealed dependent bilateral atelectasis and a mildly enlarged spleen of $14 \mathrm{~cm}$.

Results of the lumbar puncture exclude bacterial meningitis as the explanation of this patient's illness; the mildly elevated protein is nonspecific. These studies do not otherwise change the differential diagnosis.

$\overbrace{}^{2}$ The treating clinicians made a presumptive diagnosis of community-acquired pneumonia and initiated levofloxacin. He remained febrile for the next 4 days, his maximum temperature reaching $41^{\circ} \mathrm{C}$, and had intermittent hypotension with systolic blood pressure dropping to $88 \mathrm{~mm} \mathrm{Hg}$ despite intravenous fluid resuscitation. On hospital day 5 he developed worsening agitation, for which he was sedated and subsequently intubated for airway protection. The same day, vancomycin and piperacillin/tazobactam were added for presumed severe pneumonia as well as doxycycline for empiric treatment of RMSF. The patient was transferred to a tertiary care center for further care.

Supporting data for a diagnosis of pneumonia, such as pulmonary infiltrates or supplemental oxygen requirement, are lacking. Given his critical illness, broad spectrum antimicrobial coverage is indicated, and as a primary central nervous system (CNS) infection now appears unlikely, piperacillin/ tazobactam (which does not have adequate CNS penetration) and vancomycin are reasonable. Empiric treatment for RMSF is appropriate, and should have been initiated earlier in the patient's course, despite the upper Midwest being out of the typical range for this disease. Doxycycline will also provide excellent coverage for ehrlichiosis and anaplasmosis.

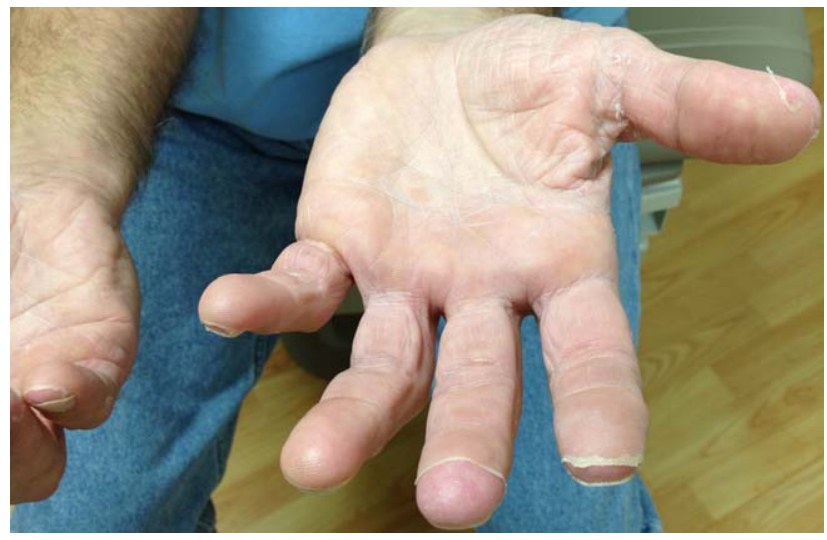

FIG. 1. Twelve days after discharge, the patient was noted to have desquamation of his palms and soles.

Given the patient's deterioration, it is important to stop and reconsider the differential diagnosis in an attempt to avoid anchoring bias and premature closure. The patient's illness is almost certainly infectious in nature, and the differential is not substantially altered by the most recent information. A skin biopsy should be performed in an attempt to secure the diagnosis.

On arrival to the tertiary care facility the patient quickly defervesced, self-extubated, and after 3 days was transitioned to doxycycline monotherapy with continued clinical improvement. At the recommendation of the infectious diseases consultant, serologies for Ehrlichia chaffeensis, Anaplasma phagocytophilum, Leptospira, Mycoplasma pneumoniae, and Rickettsia rickettsia were drawn in addition to fungal serologies for blastomycosis, coccidioidomycosis and histoplasmosis, and Legionella urinary antigen. Rapid human immunodeficiency virus testing and all cultures were negative. He was discharged home to complete a 2-week course of doxycycline for presumed RMSF.

The patient's overall course, including rapid onset of severe illness and especially the apparent dramatic response to doxycycline, make tick-borne illness very likely. Completing a course of doxycycline is certainly appropriate, typically for 7 to 14 days. The acute serologies drawn prior to discharge may well reveal the causative agent, but convalescent serology should also be obtained at the time of an outpatient follow-up visit as immunoglobulin $G$ has a delayed rise. Without hyponatremia or respiratory symptoms, Legionella seems unlikely.

Twelve days later he returned to the clinic for followup. He was overall feeling much improved and his fever, confusion, abdominal pain, and headache had resolved. $\mathrm{He}$ complained of mild fatigue, occasional myalgias, and rare nonexertional chest pain, but overall felt well. His leukocyte and platelet counts normalized, though his transaminases remained slightly elevated. His C-reactive protein decreased to $1.3 \mathrm{mg} / \mathrm{dL}$, whereas his erythrocyte sedimentation rate rose to $83 \mathrm{~mm}$. All acute serologies returned negative. Repeat convalescent serologies also returned negative. His rash had slowly faded and disappeared by his outpatient appointment; however, he was noted to have desquamation of his palms and soles (Figure 1).

The appearance of late desquamation of the palms and soles is an unexpected and important sign. Desquamation in this 
TABLE 1. 2011 Case Definition Criteria for Nonstreptococcal Toxic Shock Syndrome ${ }^{3}$

Diagnostic Criteria*

\section{Fever: Temperature $\geq 102.0^{\circ} \mathrm{F}$}

Rash: Diffuse macular erythroderma

Desquamation of rash: occurs $1-2$ weeks following rash onset

Hypotension: SBP $\leq 90 \mathrm{~mm}$ Hg for adults

Multisystem involvement, $\geq 3$ of the following:

Gl: vomiting or diarrhea at disease onset

Muscular: severe myalgias, or creatine phosphokinase $>2$ times the upper limit of normal

Mucous membranes: vaginal, oropharyngeal, or conjunctival hyperemia

Renal: BUN or Cr $>2$ times the upper limit of normal, or pyuria without evidence of infection

Hepatic: total bilirubin, AST, or ALT levels $>2$ times the upper limit of normal

Hematologic: platelets $<100,000 / \mathrm{mm}^{3}$

CNS: disorientation or altered consciousness without focal neurologic signs

Probable case: 4 out of 5 clinical criteria present

Confirmed case: 5 out of 5 clinical criteria present, or patient dies before desquamation can occur
This Case

Fever: $105.3^{\circ} \mathrm{F}$ on admission

Diffuse morbililform rash with progression to confluent erythroderma

Desquamation 12 days after discharge

Intermittent

4 organ systems definitively involved

Vomiting and abdominal pain

AST and ALT peaked at 128U/L and 94 IU/L

Platelet nadir of $80,000 / \mathrm{mm}^{3}$

Disorientation and somnolence

NOTE: Abbreviations: ALT, alanine aminotransferase; AST, aspartate aminotransferase; BUN, blood urea nitrogen; CNS, central nervous system; Cr, Creatinine; CSF, cerebrospinal fluid; Gl, gastrointestinal; SBP, systolic blood pressure. *In addition, both of the following must be fulfilled: 1) Rocky Mountain spotted fever, leptospirosis, and measles serologies negative; 2) blood and CSF cultures negative (Staphylococcus aureus or Streptococcus spp. can be positive).

pattern following an illness of this nature strongly suggests a diagnosis of staphylococcal toxic shock syndrome (TSS), and in conjunction with the negative serologies, argues that tick-borne disease is unlikely. The list of other entities that might lead to desquamation in this setting is very short, namely adult Kawasaki disease and drug reaction. The former seems reasonably excluded based on details of the case, whereas a doxycycline-related drug reaction, although not entirely implausible, seems quite unlikely as this medication was started after the onset of the initial rash. This patient most likely had staphylococcal TSS secondary to a minor and unappreciated skin lesion.

\& The patient was diagnosed with TSS, thought to be acquired through cuts and abrasions sustained while gardening. Doxycycline was discontinued and he recovered without long-term sequelae. In the following weeks, his chest pain and myalgias abated, and his palmar rash improved followed by desquamation of his soles.

\section{DISCUSSION}

TSS is a systemic illness resulting in multiorgan dysfunction. ${ }^{1}$ Infection by $S$ aureus or Streptococcus pyogenes causes TSS by stimulating maladaptive T-cell proliferation and cytokine release resulting in shock. ${ }^{1,2}$ A definitive diagnosis requires fever, a diffuse macular erythematous rash (often resembling a sunburn), with subsequent desquamation, hypotension, and involvement of at least 3 organ systems. Blood cultures, cerebrospinal cultures, and serologies for other organisms should be negative; although Staphylococcus and Streptococcus species may be isolated, they frequently are not (Table 1$).^{3}$

A rare cause of shock, TSS is most associated with a surge of menstruation-related cases linked to tampon use in young women in the 1980s. ${ }^{4}$ However, in Centers for Disease Control and Prevention (CDC) surveillance between 1987 and 1996, only $59 \%$ of the 1069 cases identified were noted to be menstruation-related, as compared to nearly $80 \%$ of all cases earlier in the decade. ${ }^{4,5}$ Today, the syndrome is more likely to present after musculoskeletal and cutaneous trauma, oropharyngeal infections, surgical procedures, and device implantation. ${ }^{1,6}$ Despite the disease's evolving epidemiology, the illness script used by physicians likely continues to focus on young women as the primary at risk population for TSS, causing physicians to neglect the diagnosis in other populations. ${ }^{1,6-9}$ Given this change in risk factors, it is imperative that clinicians rewrite their scripts and recognize the early signs of TSS in all patients to enable quick and effective treatment.

In addition to its shifting epidemiology and rarity, the diagnosis of TSS vexes clinicians for several reasons. First, TSS cannot be quickly and definitively diagnosed because 2 diagnostic criteria cannot be fulfilled during the acute illness. The disease's hallmark-a desquamative rash-occurs only if the patient survives. ${ }^{3}$ Serologies often take weeks to return, further delaying diagnosis. During this period of diagnostic delay, the illness has usually already resolved or resulted in death. In addition, the presenting symptoms of rash, fever, and shock are nonspecific. Alternative etiologies include meningococcal meningitis, which can also present dramatically as with this patient; RMSF, which can occasionally have a fulminant presentation; bacterial sepsis, usually from Staphylococcus or Streptococcus species; acute viral syndromes; and severe drug reactions. ${ }^{6,10-12}$ Palmoplantar desquamation, as in this case, can further narrow the differential as this presentation is uncommon but characteristic of TSS, RMSF, and secondary syphilis. ${ }^{11}$ Other diagnostic clues offered by the pattern of the rash may be limited by physician discomfort with diagnosing and describing rashes. Because of this lack of a definitive diagnostic test in the acute setting, it is imperative that the clinician include TSS in the differential of fever, shock, and rash, as mortality from TSS can exceed $20 \%$ in patients who are untreated. ${ }^{13}$

Treatment of TSS is straightforward once considered and includes the administration of antibiotics that cover both Staphylococcus and Streptococcus species, in addition to aggressive hydration and supportive care. ${ }^{14}$ The final critical detail in this case was the appropriate arrangement of followup. Given the patient's drastic improvement, the complicated process of arranging follow-up for a transferred patient, and the current model where the hospitalists providing inpatient care do not typically follow their patients in clinic, patients such as these can easily be lost to follow-up. Had this occurred, the desquamation would have been missed, and the patient's diagnosis would have been incomplete. 
This patient was eventually diagnosed with TSS by fulfilling all 5 CDC criteria (Table 1). ${ }^{3}$ He made a full recovery, likely aided by the administration of broadspectrum antibiotics (followed by doxycycline, which provided community-acquired methicillin-resistant $S$ aureus coverage) and his lack of serious comorbidities. This case should serve as a reminder to hospitalists that with a discerning eye, a careful assessment of the clinical facts, and appropriate follow-up, perhaps the next case of TSS can be caught red-handed.

\section{KEY POINTS}

1. When presented with a patient with fever, rash, and shock, hospitalists should consider meningococcal meningitis, RMSF bacterial sepsis, acute viral illness, severe drug reaction, and TSS.

2. TSS, caused by $S$ aureus or $S$ pyogenes, is no longer predominantly associated with tampon use. Postsurgical infection and cutaneous trauma have become important present-day risk factors.

3. The initial presentation of TSS is nonspecific. Definitive diagnosis requires proper follow-up, allowing time for infectious serologies to return negative and for the disease's hallmark desquamation to occur.

Disclosure: Nothing to report.

\section{References}

1. Low DE. Toxic shock syndrome: major advances in pathogenesis, but not treatment. Crit Care Clin. 2013;29:651-675.

2. Stevens DL. The toxic shock syndromes. Infect Dis Clin North Am. 1996;10(4):727-746.

3. Centers for Disease Control and Prevention. National Notifiable Diseases Surveillance System. Toxic shock syndrome (other than Streptococcal) (TSS) 2011 Case Definition. Available at: http://wwwn.cdc. gov/nndss/conditions/toxic-shock-syndrome-other-than-streptococcal/ case-definition/2011. Accessed June 4, 2015.

4. Centers for Disease Control and Prevention. Update: toxic-shock syndrome-United States. MMWR Morb Mortal Wkly Rep. 1983; 32(30):398-400.

5. Hajjeh RA, Reingold A, Weil A, Shutt K, Schuchat A, Perkins BA. Toxic shock syndrome in the United States: surveillance update, 1979-1996. Emerg Infect Dis. 1999;5(6):807-810.

6. Schlossberg D. Fever and rash. Infect Dis Clin North Am. 1996;10(1): 101-110.

7. DeVries AS, Lesher L, Schlievert PM, et al. Staphylococcal toxic shock syndrome 2000-2006: epidemiology, clinical features, and molecular characteristics. PLoS One. 2011;6(8):e22997.

8. Shands KN, Schmid GP, Dan BB, et al. Toxic-shock syndrome in menstruating women: association with tampon use and staphylococcus aureus and clinical features in 52 cases. N Engl J Med. 1980;303(25): 1436-1442.

9. Davis JP, Chesney PJ, Wand PJ, LaVenture M. Toxic-shock syndrome-epidemiologic features, recurrence, risk factors, and prevention. N Engl J Med. 1980;303:1429-1435.

10. McKinnon HD, Howard T. Evaluating the febrile patient with a rash. Am Fam Physician. 2000;62(4):804-816.

11. Herzer CM. Toxic shock syndrome: broadening the differential diagnosis. I Am Board Fam Pract. 2001;14(2):131-136.

12. Adjemian JZ, Krebs J, Mandel E, McQuiston J. Spatial clustering by disease severity among reported Rocky Mountain spotted fever cases in the United States, 2001-2005. Am J Trop Med Hyg. 2009;80(1): $72-77$.

13. Descloux E, Perpoint T, Ferry T, et al. One in five mortality in nonmenstrual toxic shock syndrome versus no mortality in menstrual cases in a balanced French series of 55 cases. Eur J Clin Microbio Infect Dis. 2008;27(1):37-43.

14. Lappin E, Ferguson AJ. Gram-positive toxic shock syndromes. Lancet Infect Dis. 2009;9(5):281-290. 